\title{
EFFICACY OF PHYSICAL AND CHEMICAL TREATMENT OF BROILER RATION TO REDUCE THE TOXICITY OF T- 2 TOXIN IN BROILER CHICKEN.
}

\author{
Kamel, H. H. \\ Department of Clinical Pathology, Fac. of Vet. Med., Beni-Suef Univ.
}

\begin{abstract}
Mycotoxicosis is one of the most important problems affecting poultry industry. T-2 toxin is an example of the Omycotoxins which affects poultry productivity. The present research was designed to study the effect of T-2 toxin on the body weight, hemogram, serum biochemistry, histopatholgical changes and toxin residues in muscles of broilers chickens. In addition the study included the possibility of reduction of the toxic effects of this toxin by the either physical (dryness of the ration at $200^{\circ} \mathrm{C}$ for 1 hour) or chemical means (addition of Hydrated Sodium Calcium Aluminiosilicate, HSCA). One hundred and fifty Hubbard broiler chicks of one day old were randomly allotted into five groups of 30 each. The $1^{\text {st }}$ group received ration mixed with sterile yeast extract sucrose broth $37.5 \mathrm{ml} / \mathrm{kg}$ ration and considerd as a control group. The $2^{\text {nd }}$ group received the same amount of the previous broth + HSCAS in a dose of $2 \mathrm{~kg} / \mathrm{ton}$ ration. The $3^{\text {rd }}$ group received ration mixed with $T-2$ toxin ( $3 \mathrm{mgT}-2$ contained in $37.5 \mathrm{ml}$ of yeast extract sucrose broth $/ \mathrm{kg}$ ration). The $4^{\text {th }}$ group received the same dose of T-2 toxin in a ration treated physically by dryness at 200 ${ }^{\circ} \mathrm{C}$ for 1 hour while the $5^{\text {th }}$ group received the same amount of $T-2$ toxin in a ration treated chemically by addition of HSCAS at a dose of $2 \mathrm{~kg} / \mathrm{ton}$ ration for 6 weeks. At the end of every week, body weight was recorded and blood samples were collected for hematological and serum biochemical determination. Tissue specimens from liver, kidney, heart, and intestine were taken for histopathological examination weekly for 6 weeks and muscle specimens were taken at the end of the $6^{\text {th }}$ week for detection of T-2 toxin residues. Results of groups 3 and 4 received T-2 toxin, revealed reduction in the body
\end{abstract}


weight, significant decrease in $\mathrm{Hb}, P C V$ and RBCs count and significant decrease in WBCs count accompanied with heteropenia and lymphocytosis from the $3^{\text {rd }}$ week till the end of the experiment. Levels of blood glucose, uric acid and BUN and activity of ALT, AST and GGT were significantly increased. Values of total proteins, albumin, globulin and cholesterol were significantly decreased only in the $3^{\text {rd }}$ group. Histopathological examination revealed harmful effects of $T-2$ on the liver, kidney, heart and intestine. Measurement of the toxin residues in muscles of toxicated chicken revealed presence of the toxin in all muscles with different amounts. Results showed that the above described changes were insignificant in other treated groups. This study showed a beneficial effects of the physical and chemical treatment of the ration in reducing the toxic effects of T-2 toxin. Chemical treatment was founded better than physical treatment.

\section{INTRODUCTION}

Contamination of animal feed with mycotoxins represents a worldwide problem. for farmer. Mycotoxins are structurally diverse secondary metabolites of fungi that grow on feed stuffs consumed by animals and human. Mycotoxin-containing feed can cause serious disease in animals (mycotoxicosis) which may lead to death, thus causeing substantial economic losses. Cereal plants may be contaminated by mycotoxins through fungi growing as pathogens on plants, or fungi growing saprophytically on stored cereals (Council for Agricultural Science and Technology, 1988). Among the most frequently mentioned fungi are members of the Fusarium genera which are widely distributed in cereal grains and feed stuffs in Egypt (El-Hamaky, 2001). The most important Fusarium mycotoxins that can frequently occur at biologically significant concentrations in cereals are trichothecenes (deoxynivalenol, nivalenol and T-2 toxin ), zearalenone and fumonisins (Richard, 2003). T-2 toxin has a special concern in poultry industry due to its potential for major health problems and economic losses. The toxin causes acute $\overline{\text { Kafrelsheikh Vet. Med. J. Vol. } 5 \text { No. } 1 \text { (2007) }}$ 
mortality (Corrier, 1991), reduced body weight (Bailey et al., 1998) and reduced egg production (Grizzle et al., 2005). The importance of T-2 mycotoxicosis is not restricted only to poultry industry but also it extended to public health as the toxin constitutes a public health hazard. T-2 toxin caused a human disease known as Alimentary Toxic Aleukia (ATA) which was responsible for mortality rate of $60 \%$ of the population affected in Russia (Joffe, 1971). Moreover, direct contact with the toxin caused dermatitis (Beasley, 1989), T-2 toxin had a radiomimetic cellular damage in the haematopoietic and lymphopoietic systems (Hayes et al., 1980) and had an embryotoxic effects (Janovitz, 1988).

Extensive research have been conducted to counter mycotoxins by physical, chemical, nutritional or biological approaches (Raju and Devegowda, 2000). Therefore, the purpose of the following research was to study the blood and histopathological changes in broilers exposed to T-2 toxin, detection of the mycotoxin residues in muscles of experimentally toxinated chickens and evaluation of the efficacy of physical and chemical treatment of the broiler ration to reduce the toxicity of T-2 toxin.

\section{MATERIAL AND METHODS}

I- Material: 1-Experimental birds: One hundred and fifty, one day old hubbard broiler chicks obtained from Arab Poultry Breeders Company, Giza were used. They were fed on balanced commercial ration (EL-Kahira) with free access to food and water. Chicken were left for 10 days before start of the experiment for acclimatization.

2- T-2 toxin: The toxin was kindly supplied from Animal Health Research Institute. T-2 toxin a naturally occurring mycotoxin produced by fusarium species, which is a 3 hydroxy 4,15 diacetoxy- 8 (3-methylbutryloxy), 12, 13 epoxy tricothec-9-ene metabolite (krishnamoorthy et al., 2006). Each $37.5 \mathrm{ml}$ of $37.5 \mathrm{ml}$ of yeast extract sucrose broth contained $3 \mathrm{mg}$ of the toxin. 
3- Chemicals: Hydrated Sodium Calcium Aluminiosilicate 100\% (HSCAS) was obtained from Sat Pharma Vet. Company. It was given at a dose of $2 \mathrm{~kg} /$ ton ration (Casarin et al., 2006).

4- Heat treated ration: The commercial ration (EL-Kahira) was mixed with $\mathrm{T}-2$ toxin in a dose of $3 \mathrm{mg} / \mathrm{kg}$ ration and treated physically by dry heat at $200^{\circ} \mathrm{C}$ for one hour daily for 6 weeks. (Ragheb, 1994).

5- Diagnostic Kits: Determination of serum biochemical constituents was performed by using kits from Scientific Co for Chemical Industries (cholesterol, BUN and uric acid), Cromatest Linear Chemicals (total protein, albumin and glucose) and Diamond Diagnostics Company (ALT, AST and GGT).

II- Methods: 1-Experimental design: Birds were divided into 5 equal groups: group I was kept as control and received free Yeast Extract Sucrose Broth ( $37.5 \mathrm{ml} / \mathrm{kg}$ ration) daily for 6 weeks, group II received the same dose of the previous broth plus HSCAS at a dose of $2 \mathrm{~kg} / \mathrm{ton}$ ration daily for 6 weeks, group III received T-2 toxin at a dose of 3 $\mathrm{mg} / \mathrm{kg}$ ration (Raju and Devegowda, 2000) daily for 6 weeks, group IV received the ration containing the same dose of T-2 toxin but treated physically by heat, while group $\mathrm{V}$ received the ration containing the same dose of T-2 toxin and treated chemically by addition of HSCAS. Clinical signs and mortalities were recorded, the chicks were weighted weekly.

2-Sampling and analyses: Blood samples were taken from individuals of each group weekly for 6 weeks. Blood was divided into 2 portions, one for performing the hemogram (Jain, 1986) and the other for serum separation for determination of ALT and AST (Reitman and Frankel, 1957), GGT (Whitfield et al., 1973), glucose (Trinder, 1969), total protein (Henry et al., 1974), serum albumin (Beng and $\overline{\text { Kafrelsheikh Vet. Med. J. Vol. } 5 \text { No. } 1 \text { (2007) }}$ 
Lim, 1973), cholesterol (Henry et al., 1974), serum BUN (Reale and Croft, 1961) and uric acid (Young, 2001). Tissue samples from liver, kidney, heart, and intestine were taken at the end of the $6^{\text {th }}$ week and kept in formalin 10\% for histopathological examination (Bancroft and Stevens, 1996). Muscle specimens also were obtained from T-2 toxin exposed birds (of groups III, IV and V) for detection of T-2 toxin residues (Visconti and Mirocha, 1985).

Statistical analysis: Data were compared across groups using one way analysis of variance (ANOVA) F-test according to Snedecor and Cochran, (1967).

\section{RESULTS}

1- Clinical signs, body weight and mortality rate: Severe depression, ruffled feathers and reduction in feed consumption and body weight were the most characteristic signs in the 3rd group received the toxin only. These signs were mild in the 4th group (received T-2 toxin ration treated physically) and the 5 th group (received T-2 toxin ration treated chemically) (table, 1). At the end of the 2 nd week of the experiment, mortality rate was $6 \%$ in the 3 rd group, and $2 \%$ in both the 4 th and 5 th groups.

2- Hemogram: Erythrogram showed significant decrease in $\mathrm{Hb}, \mathrm{PCV}$ and RBCs count in the $3^{\text {rd }}$ group (received T-2 toxin) and the $4^{\text {th }}$ group (received T-2 toxin and treated physically) from the $3^{\text {rd }}$ week till the end of the experiment (table, 2). The developed anemia was of the macrocytic hypochromic type. Leucogram showed significant leucopenia accompanied with heteropenia and lymphocytosis only in the $3^{\text {rd }}$ group from the $2^{\text {nd }}$ week till the end of the experiment (table, 3). 
3- Serum biochemical results:- The obtained data from the $3^{\text {rd }}$ group which received T-2 toxin and the $4^{\text {th }}$ group that received heat - treated T-2 toxin ration denoted significant increase in the activities of aminotransferases. This elevation was observed from the end of the $2^{\text {nd }}$ week till the end of the experiment. GGT activities showed no significant differences along the experiment. Levels of serum BUN and uric acid revealed significant elevation in group III from the $3^{\text {rd }}$ week till the end of the experiment (table, 4). Levels of glucose showed significant increase in the first two weeks, then showed significant decrease from the third week till the end of the experiment. Total protein, albumin, globulin and cholesterol values were significantly decreased from the $1^{\text {st }}$ week till the end of the experiment in the T-2 toxin group. Values in other experimental groups showed no significant changes (table, 5).

\section{4-Histopathological examination:}

i- Examination of groupII received HSCAS did not show any pathological alterations.

\section{ii- Results of histopathological examination of group III received T-2} toxin:

Liver: Grossly, the liver appeared enlarged with the presence of congested and /or pale areas at 1,3,4,5, and 6 weeks. Microscopically, the liver showed mild hepatocellular degeneration with dilatation and congestion of the central veins. There was multifocal leucocytic infiltration, mainly of mononuclear cells, within the hepatic parenchyma. Previous findings were observed 1, 2, 3, and 4 weeks of the experiment (fig.1). 
Kidneys: Grossly, the kidneys, 5 weeks of the experiment were enlarged and contained hemorrhagic spots. Microscopically, the renal epithelial cells of the proximal and distal convoluted tubules showed marked pyknosis of their nuclei. Apoptosis was found in the most of the renal tubules of this group (fig.2). The glomeruli appeared hyperplastic with widening of Bowman's capsules specially 1, 2, and 3 weeks post T-2 toxin exposure. In some cases, congestion of the intertubular veins was observed with hemorrhage in some areas specially in the cortical tissue after 3 and 6 weeks of the experiment. Renal calcinosis was also found within some renal tubules particularly 4 and 6 weeks post exposure. The later change was associated with severe necrotic changes and sloughing of the epithelial cells lining the affected tubules. Focal interstitial nephritis was recorded 5 weeks post exposure. Focal or diffuse leucocytic infiltration was observed in between the glandular pits.

Heart: The heart appeared congested with the presence of hemorrhagic spots after 3 weeks of T-2 exposure.. Myocardial degeneration (vacuolation) was seen in birds exposed to the toxin for 1 and 2 weeks. Clear empty vacuoles were found within the cytoplasm of muscle cells. Slight congestion was observed at 2, 3, and 4 weeks post exposure (fig.3). Focal leucocytic aggregations were demonstrated within the myocardial muscles after one week of exposure. Lymphocytes and macrophages were the main component of these cellular infiltrations.

Intestine: Grossly, the intestine revealed catarrhal entritis in all birds with the presence of peticheal hemorrhages on the serosal surface of the intestine. Histopathologically, entritis was seen among birds exposed to T-2 toxin regardless to exposure time. Severe 
degeneration, necrosis, and detaching of epithelial cells lining the villi and crypts were found in most cases. In some birds, the intestinal lumen was dilated and filled with cellular core comprising of desquamated epithelial cells and mononuclear cells. Mononuclear cell infiltration was found within and around degenerated crypts and in the lamina propria of most chickens. Mild to severe congestion was observed in submucosal and muscular blood vessels specially in the cases slaughtered 1 and 2 weeks post exposure.

\section{iii- Results of histopathological examination of group IV received T- 2 toxin ration and treated by heat:}

Liver: Grossly, liver was congestion 1, 2 and 3 weeks post exposure. After 5 weeks the liver appeared yellowish in color. Histopathologically, nuclei of hepatic cells appeared pyknotic 1 week post exposure. Dilatation and congestion of central veins and focal leucocytic infiltrations were observed in chickens slaughtered 1 and 3 weeks after exposure to the toxin (fig. 4).

Kidneys: Mild gross pathological alterations were seen in the form of congestion and mild enlargement specially 5 and 6 weeks post exposure. Microscopically, the kidneys showed degenerative changes (vacuolation) in most cases specially in the renal cortex. Nuclear condensation was found in the renal epithelium (fig.5) of chickens in all weeks except the first. Renal epithelial cells showed apoptotic changes in a large number of the tubules specially at the $3^{\text {rd }}$ and $6^{\text {th }}$ weeks. At the $4^{\text {th }}$ and $6^{\text {th }}$ weeks post exposure, focal leucocytic infiltration was recorded in the interstitial areas with congestion of blood vessels. 
Heart: Very mild degenerative changes were observed in the heart of some birds slaughtered after 3,5 and 6 weeks of the experiment.

Intestine: At the end of the $4^{\text {th }}$ week of the experiment, catarrhal enteritis with distension and congestion of the small intestine were noticed in most cases. Microscopically, moderate to severe enteritis was found in the intestine of most birds (fig.6). Severe enteritis appeared specially after 1 and 5 weeks of exposure with the presence of inflammatory exudates within the intestinal lumen. The surface epithelium of the villi and upper part of crypts showed degenerative changes and mild necrosis. The submucosal area was infiltrated with mononuclear cells.

\section{iv- Results of histopathological examination of group $\mathrm{V}$ received T-2 toxin ration chemically treated:}

Liver: Areas of congestion appeared in the liver 1, 2 and 3 weeks after exposure. The liver appeared grossly enlarged with the presence of pale batches after 5 and 6 weeks. Microscopically, the hepatocytes showed mild signs of degeneration. Focal leucocytic infiltration (fig.7) and mild congestion were seen in the hepatic parenchyma of chicken slaughtered 3 - 6 weeks post exposure.

Kidneys: In most cases, the renal epithelial cells showed mild degree of nuclear condensation specially after 3, 4, and 5 weeks(fig.8). Mild apoptosis was seen 6 weeks post exposure. The intertubular blood vessels were dilated and congested in most chickens. Focal interstitial nepheritis was seen 4 and 6 weeks post exposure.

Heart: Grossly, hemorrhagic spots were found after 5 weeks .Microscopically, most chickens showed very mild degenerative changes of myocardial muscles and congestion of blood vessels. Only one chicken had fibrinous pericarditis and another one showed focal leucocytic infiltration 5 weeks post exposure. (fig.9). 
Intestine: Microscopically, mild enteritis was noticed in most cases (fig.10). The submucosal area was infiltrated with mononuclear cells.

5- Measurement of T-2 toxin residues: Results revealed presence of toxin residues in chicken meat with different amounts as shown in table (6). The highest concentration was found in the group received $\mathrm{T}-2$ toxin, followed by the group received T-2 toxin ration physically treated by heat. The lowest concentration of residues was recorded in the group received T- 2 toxin ration chemically treated by HSCAS. Muscles of chickens of the control group were free from T-2 toxin residues.

Table (1): Body weight values in grams in different experimental groups of broiler chickens.

\begin{tabular}{|c|c|c|c|c|c|c|}
\hline Time & 1 & 2 & 3 & 4 & 5 & 6 \\
\hline I & $\begin{array}{r}103.33 \\
\pm 3.40 \\
\end{array}$ & $\begin{array}{r}202.50 \\
\pm 4.30 \\
\end{array}$ & $\begin{array}{l}506.66 \\
\pm 5.00 \\
\end{array}$ & $\begin{array}{l}606.66 \\
\pm 5.21 \\
\end{array}$ & $\begin{array}{r}916.66 \\
\pm 7.00 \\
\end{array}$ & $\begin{array}{c}1233.33 \\
\pm 3.90 \\
\end{array}$ \\
\hline II & $\begin{array}{l}82.50 \\
\pm 2.50\end{array}$ & $\begin{array}{l}185.00 \\
\pm 8.80\end{array}$ & $\begin{array}{l}466.66 \\
\pm 6.00\end{array}$ & $\begin{array}{l}600.00 \\
\pm 6.44\end{array}$ & $\begin{array}{l}906.66 \\
\pm 6.80\end{array}$ & $\begin{array}{c}1366.66 \\
\pm 3.90\end{array}$ \\
\hline III & $\begin{array}{l}93.75 \\
\pm 2.40\end{array}$ & $\begin{array}{l}147.66 \\
\pm 2.28 *\end{array}$ & $\begin{array}{c}366.66 \\
\pm 6.80 * *\end{array}$ & $\begin{array}{c}446.66 \\
\pm 6.80 * *\end{array}$ & $\begin{array}{c}596.00 \\
\pm 4.06^{* *}\end{array}$ & $\begin{array}{c}666.66 \\
\pm 3.90^{* *}\end{array}$ \\
\hline IV & $\begin{array}{l}96.25 \\
\pm 2.40\end{array}$ & $\begin{array}{r}146.66 \\
\pm 6.80 *\end{array}$ & $\begin{array}{c}480.00 \\
\pm 5.80 *\end{array}$ & $\begin{array}{c}560.00 \\
\pm 5.66^{*}\end{array}$ & $\begin{array}{c}826.66 \\
\pm 27.20^{*}\end{array}$ & $\begin{array}{l}1166.66 \\
\pm 3.90 *\end{array}$ \\
\hline $\mathrm{V}$ & $\begin{array}{l}105.00 \\
\pm 5.90\end{array}$ & $\begin{array}{l}195.00 \\
\pm 4.50\end{array}$ & $\begin{array}{l}500.00 \\
\pm 4.80\end{array}$ & $\begin{array}{l}606.66 \\
\pm 5.30\end{array}$ & $\begin{array}{l}846.66 \\
\pm 4.92\end{array}$ & $\begin{array}{c}1250.00 \\
\pm 8.90\end{array}$ \\
\hline
\end{tabular}

Group I Normal control.

Group II Normal ration + HSCAS.

Group III T-2 toxin ration.

Group IV T-2 toxin ration physically treated.

Group V T-2 toxin ration chemically treated.

* Significantly different from normal control group, $\mathrm{p}<0.05$.

** Highly significantly different from normal control group, $\mathrm{p}<0.001$.

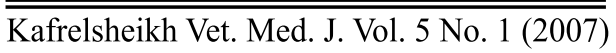


Efficacy Of Physical And Chemical Treatment Of Broiler Ration To ...

Table (2 ): Hemogram in different experimental groups of broiler chickens.

\begin{tabular}{|c|c|c|c|c|c|c|c|}
\hline$\sum_{0}^{0}$ & 을 & $\begin{array}{c}\text { Hb } \\
\text { (g/dl.) }\end{array}$ & $\begin{array}{c}\text { P.C.V } \\
(\%)\end{array}$ & $\begin{array}{c}\text { R.B.Cs } \\
\left(\mathbf{x} 10^{6} / \mu \mathrm{l} .\right)\end{array}$ & $\begin{array}{c}\text { MCV } \\
\text { (fl.) }\end{array}$ & $\begin{array}{c}\text { MCH } \\
\text { (Pg.) }\end{array}$ & $\begin{array}{c}\text { МСHC } \\
(\%)\end{array}$ \\
\hline W1 & $\begin{array}{l}\text { I } \\
\text { II } \\
\text { III } \\
\text { IV } \\
\text { V }\end{array}$ & $\begin{array}{c}8.00 \pm 0.10 \\
8.18 \pm 0.66 \\
8.50 \pm 0.50 \\
8.66 \pm 0.80 \\
8.93 \pm 1.10\end{array}$ & $\begin{array}{l}28.13 \pm 2.06 \\
28.33 \pm 2.90 \\
27.00 \pm 0.60 \\
29.00 \pm 0.80 \\
29.30 \pm 0.85\end{array}$ & $\begin{array}{l}3.46 \pm 0.34 \\
3.41 \pm 0.37 \\
3.28 \pm 0.46 \\
3.54 \pm 0.32 \\
3.36 \pm 0.26\end{array}$ & $\begin{array}{c}78.50 \pm 3.80 \\
77.90 \pm 3.00 \\
73.20 \pm 2.90 \\
77.00 \pm 2.90 \\
78.80 \pm 3.50\end{array}$ & $\begin{array}{l}24.06 \pm 6.60 \\
24.52 \pm 2.40 \\
25.10 \pm 1.70 \\
25.00 \pm 4.50 \\
24.10 \pm 4.90\end{array}$ & $\begin{array}{l}28.80 \pm 0.2 . \\
29.90 \pm 1.80 \\
28.10 \pm 1.20 \\
28.60 \pm 1.30 \\
29.00 \pm 0.50\end{array}$ \\
\hline W2 & $\begin{array}{l}\text { I } \\
\text { II } \\
\text { III } \\
\text { IV } \\
\text { V }\end{array}$ & $\begin{array}{l}9.40 \pm 0.60 \\
9.40 \pm 0.40 \\
9.30 \pm 1.06 \\
9.85 \pm 0.67 \\
9.66 \pm 0.72\end{array}$ & $\begin{array}{l}29.80 \pm 0.40 \\
30.00 \pm 1.50 \\
29.66 \pm 0.70 \\
30.13 \pm 0.70 \\
29.00 \pm 1.00\end{array}$ & $\begin{array}{l}3.74 \pm 0.26 \\
3.35 \pm 0.08 \\
3.46 \pm 0.07 \\
3.69 \pm 0.13 \\
3.39 \pm 0.20\end{array}$ & $\begin{array}{c}79.00 \pm 2.60 \\
77.20 \pm 3.30 \\
78.70 \pm 0.90 \\
80.80 \pm 0.60 \\
78.60 \pm 4.20\end{array}$ & $\begin{array}{l}25.90 \pm 3.60 \\
24.80 \pm 4.30 \\
24.50 \pm 1.50 \\
25.00 \pm 5.60 \\
24.50 \pm 7.30\end{array}$ & $\begin{array}{c}30.40 \pm 20 \\
31.10 \pm 0.80 \\
31.30 \pm 2.40 \\
30.80 \pm 0.90 \\
31.40 \pm 2.80\end{array}$ \\
\hline W3 & $\begin{array}{c}\text { I } \\
\text { II } \\
\text { III } \\
\text { IV } \\
\text { V }\end{array}$ & $\begin{array}{c}9.16 \pm 1.30 \\
9.44 \pm 1.05 \\
7.68 \pm 0.69 * \\
8.50 \pm 1.30 * \\
9.00 \pm 0.40\end{array}$ & $\begin{array}{c}30.40 \pm 0.80 \\
31.80 \pm 1.90 \\
29.60 \pm 1.50^{*} \\
28.66 \pm 1.20^{*} \\
31.80 \pm 0.50\end{array}$ & $\begin{array}{l}3.56 \pm 0.10 \\
3.83 \pm 0.34 \\
3.07 \pm 0.08 \\
3.39 \pm 0.20 \\
3.25 \pm 0.35\end{array}$ & $\begin{array}{c}87.60 \pm 3.40 \\
88.10 \pm 3.30 \\
97.10 \pm 6.60^{*} \\
98.90 \pm 2.10^{*} \\
87.70 \pm 3.1\end{array}$ & $\begin{array}{c}24.8 \pm 4.90 \\
24.3 \pm 3.10 \\
25.00 \pm 4.10 \\
24.60 \pm 5.70 \\
26.90 \pm 3.80\end{array}$ & $\begin{array}{c}31.20 \pm 3.00 \\
30.90 \pm 2.40 \\
24.60 \pm 0.10^{*} \\
28.05 \pm 2.70 * \\
30.00 \pm 1.60\end{array}$ \\
\hline W4 & $\begin{array}{c}\text { I } \\
\text { II } \\
\text { III } \\
\text { IV } \\
\text { V }\end{array}$ & $\begin{array}{c}9.96 \pm 2.30 \\
9.26 \pm 0.80 \\
8.56 \pm 1.30^{*} \\
8.10 \pm 1.20^{*} \\
10.17 \pm 2.30\end{array}$ & $\begin{array}{c}33.75 \pm 1.90 \\
32.80 \pm 1.70 \\
27.60 \pm 1.80^{*} \\
29.30 \pm 0.80^{*} \\
32.00 \pm 1.20\end{array}$ & $\begin{array}{c}3.65 \pm 0.26 \\
3.57 \pm 0.40 \\
2.50 \pm 0.38 * \\
3.34 \pm 0.40 \\
3.26 \pm 0.50\end{array}$ & $\begin{array}{c}89.50 \pm 4.60 \\
85.20 \pm 0.70 \\
102.8 \pm 1.6^{*} \\
90.90 \pm 2.10^{*} \\
88.80 \pm 3.20\end{array}$ & $\begin{array}{l}25.50 \pm 5.50 \\
24.60 \pm 4.00 \\
24.20 \pm 4.50 \\
24.20 \pm 3.00 \\
25.30 \pm 4.30\end{array}$ & $\begin{array}{c}33.50 \pm 0.90 \\
34.40 \pm 1.50 \\
29.50 \pm 1.40 * \\
32.50 \pm 3.80 \\
27.90 \pm 2.50\end{array}$ \\
\hline W5 & $\begin{array}{l}\text { I } \\
\text { II } \\
\text { III } \\
\text { IV } \\
\text { V }\end{array}$ & $\begin{array}{c}8.70 \pm 1.60 \\
9.4 \pm 1.50 \\
6.25 \pm 0.1^{*} \\
8.90 \pm 0.70 \\
7.08 \pm 0.70^{*}\end{array}$ & $\begin{array}{c}31.00 \pm 0.85 \\
30.60 \pm 0.83 \\
24.30 \pm 0.20 * * \\
30.50 \pm 1.50 \\
28.66 \pm 0.98^{*}\end{array}$ & $\begin{array}{c}3.94 \pm 0.50 \\
3.77 \pm 0.20 \\
2.57 \pm 0.24 * \\
3.47 \pm 0.29 \\
3.47 \pm 0.28\end{array}$ & $\begin{array}{c}89.30 \pm 3.00 \\
89.03 \pm 2.50 \\
99.50 \pm 4.10^{*} \\
82.50 \pm 3.10 \\
86.50 \pm 2.80\end{array}$ & $\begin{array}{l}25.40 \pm 3.20 \\
24.10 \pm 3.90 \\
23.50 \pm 4.40 \\
24.70 \pm 4.90 \\
23.90 \pm 4.50\end{array}$ & $\begin{array}{l}30.50 \pm 2.00 \\
31.90 \pm 1.50 \\
25.40 \pm 0.40 \\
31.80 \pm 0.60 \\
25.90 \pm 0.80\end{array}$ \\
\hline W6 & $\begin{array}{c}\text { I } \\
\text { II } \\
\text { III } \\
\text { IV } \\
\text { V }\end{array}$ & $\begin{array}{c}8.75 \pm 0.98 \\
8.43 \pm 0.60 \\
7.45 \pm 0.05^{*} \\
7.87 \pm 0.53^{*} \\
8.90 \pm 0.51\end{array}$ & $\begin{array}{c}30.50 \pm 0.30 \\
30.30 \pm 1.20 \\
27.30 \pm 0.30 * \\
28.90 \pm 1.00 * \\
29.40 \pm 0.70\end{array}$ & $\begin{array}{c}3.70 \pm 0.303 .34 \\
\pm 0.30 \\
2.15 \pm 0.16^{*} \\
3.40 \pm 0.10 \\
3.85 \pm 0.25\end{array}$ & $\begin{array}{c}89.30 \pm 3.30 \\
93.70 \pm 2.80 \\
108.50 \pm 5.50 * \\
85.20 \pm 2.60 \\
81.20 \pm 1.00\end{array}$ & $\begin{array}{l}24.60 \pm 3.50 \\
24.30 \pm 4.00 \\
24.90 \pm 3.50 \\
24.40 \pm 4.80 \\
24.20 \pm 2.10\end{array}$ & $\begin{array}{c}30.70 \pm 1.40 \\
30.90 \pm 2.70 \\
25.30 \pm 0.10^{*} \\
25.80 \pm 1.30 * \\
29.30 \pm 1.4\end{array}$ \\
\hline
\end{tabular}

Group I Normal control.

Group II Normal ration + HSCAS.

Group III T-2 toxin ration.

Group IV T-2 toxin ration physically treated.

Group V T-2 toxin ration chemically treated.

* Significantly different from normal control group, $\mathrm{p}<0.05$.

** Highly significantly different from normal control group, $\mathrm{p}<0.001$.

$\overline{\text { Kafrelsheikh Vet. Med. J. Vol. } 5 \text { No. } 1 \text { (2007) }}$ 
Table (3): leukogram in different experimental groups of broiler chickens.

\begin{tabular}{|c|c|c|c|c|c|c|}
\hline \multirow{2}{*}{$\begin{array}{l}\text { Time/ } \\
\text { week }\end{array}$} & \multirow{2}{*}{ Group } & \multirow{2}{*}{$\begin{array}{l}\text { W.B.Cs } \\
\text { x10 } 10^{3} / \mu l .\end{array}$} & \multicolumn{4}{|c|}{ Differential leucocytic count $\times 10^{3} / \mu \mathrm{l}$. } \\
\hline & & & Heterophiles & Lymphocytes & Monocytes & Esinophiles \\
\hline \multirow{5}{*}{$1 \mathrm{w}$} & $\mathrm{I}$ & $12.75 \pm 0.25$ & $6.16 \pm 0.41$ & $4.36 \pm 0.62$ & $2.92 \pm 0.06$ & $0.04 \pm 0.01$ \\
\hline & II & $12.50 \pm 0.65$ & $6.03 \pm 0.21$ & $4.48 \pm 0.20$ & $2.91 \pm 0.06$ & $0.08 \pm 0.02$ \\
\hline & III & $11.60 \pm 0.2$ & $6.20 \pm 0.23$ & $4.31 \pm 0.90$ & $2.82 \pm 0.04$ & $0.04 \pm 0.01$ \\
\hline & IV & $13.10 \pm 0.25$ & $6.10 \pm 0.41$ & $4.33 \pm 0.20$ & $2.06 \pm 0.07$ & $0.04 \pm 0.01$ \\
\hline & $\mathrm{V}$ & $12.20 \pm 0.31$ & $6.11 \pm 0.21$ & $4.33 \pm 0.41$ & $2.92 \pm 0.05$ & $0.06 \pm 0.01$ \\
\hline \multirow{5}{*}{$2 w$} & $\mathrm{I}$ & $12.01 \pm 0.41$ & $6.30 \pm 0.51$ & $4.33 \pm 0.30$ & $2.06 \pm 0.01$ & $0.46 \pm 0.06$ \\
\hline & II & $11.01 \pm 0.25$ & $6.60 \pm 0.43$ & $4.51 \pm 0.21$ & $2.30 \pm 0.01$ & $0.26 \pm 0.03$ \\
\hline & III & $8.33 \pm 0.34 *$ & $3.11 \pm 0.62 *$ & $8.33 \pm 0.61 *$ & $2.41 \pm 0.01$ & $0.22 \pm 0.02$ \\
\hline & IV & $13.80 \pm 0.61$ & $6.12 \pm 0.41$ & $4.23 \pm 0.40$ & $2.06 \pm 0.02$ & $0.28 \pm 0.03$ \\
\hline & $\mathrm{V}$ & $12.75 \pm 0.20$ & $6.21 \pm 0.21$ & $4.13 \pm 0.23$ & $2.96 \pm 0.02$ & $0.42 \pm 0.01$ \\
\hline \multirow{5}{*}{ 3w } & $\mathbf{I}$ & $12.40 \pm 0.25$ & $6.51 \pm 0.41$ & $4.23 \pm 0.70$ & $1.33 \pm 0.90$ & $0.16 \pm 0.01$ \\
\hline & II & $13.54 \pm 0.51$ & $5.41 \pm 0.61$ & $4.53 \pm 0.21$ & $1.11 \pm 0.60$ & $0.13 \pm 0.03$ \\
\hline & III & $8.61 \pm 0.32 *$ & $3.66 \pm 0.24 *$ & $7.64 \pm 0.31 *$ & $1.63 \pm 0.71$ & $0.09 \pm 0.01$ \\
\hline & IV & $13.65 \pm 0.25$ & $6.70 \pm 0.45$ & $471 \pm 0.61$ & $1.33 \pm 0.51$ & $0.08 \pm 0.02$ \\
\hline & $\mathbf{V}$ & $12.52 \pm 0.37$ & $6.22 \pm 0.51$ & $4.80 \pm 0.8$ & $1.30 \pm 0.12$ & $0.11 \pm 0.01$ \\
\hline \multirow{5}{*}{$4 w$} & I & $16.23 \pm 0.53$ & $6.05 \pm 0.24$ & $4.07 \pm 0.11$ & $1.25 \pm 0.21$ & $0.11 \pm 0.01$ \\
\hline & II & $15.22 \pm 0.6$ & $6.13 \pm 0.21$ & $4.33 \pm 0.20$ & $1.15 \pm 0.61$ & $0.08 \pm 0.03$ \\
\hline & III & $9.50 \pm 0.44 *$ & $3.51 \pm 0.01 *$ & $7.96 \pm 0.70 *$ & $1.21 \pm 0.43$ & $0.15 \pm 0.02$ \\
\hline & IV & $16.50 \pm 0.42$ & $6.5 \pm 0.61$ & $4.03 \pm 0.09$ & $1.35 \pm 0.71$ & $0.13 \pm 0.03$ \\
\hline & $\mathbf{V}$ & $16.80 \pm 0.51$ & $6.10 \pm 0.22$ & $4.33 \pm 0.51$ & $1.13 \pm 0.32$ & $0.26 \pm 0.03$ \\
\hline \multirow{5}{*}{$5 w$} & $\bar{I}$ & $16.66 \pm 0.81$ & $5.5 \pm 0.32$ & $4.06 \pm 0.21$ & $0.91 \pm 0.10$ & $0.15 \pm 0.05$ \\
\hline & II & $15.4 \pm 0.40$ & $5.75 \pm 0.84$ & $4.33 \pm 0.42$ & $1.06 \pm 0.07$ & $0.08 \pm 0.03$ \\
\hline & III & $8.66 \pm 0.30^{*}$ & $2.40 \pm 0.44^{*}$ & $8.66 \pm 0.34 *$ & $0.81 \pm 0.06$ & $0.11 \pm 0.05$ \\
\hline & IV & $15.21 \pm 0.51$ & $6.45 \pm 0.42$ & $4.33 \pm 0.12$ & $1.66 \pm 0.71$ & $0.09 \pm 0.03$ \\
\hline & $\mathbf{V}$ & $16.01 \pm 0.54$ & $6.81 \pm 0.61$ & $4.81 \pm 0.07$ & $0.93 \pm 0.7$ & $0.12 \pm 0.01$ \\
\hline \multirow{5}{*}{$6 w$} & I & $16.40 \pm 0.41$ & $6.50 \pm 0.51$ & $4.25 \pm 0.21$ & $0.45 \pm 0.08$ & $0.09 \pm 0.01$ \\
\hline & II & $16.33 \pm 0.32$ & $6.31 \pm 0.16$ & $4.16 \pm 0.18$ & $0.75 \pm 0.20$ & $0.07 \pm 0.02$ \\
\hline & III & $9.50 \pm 0.51 *$ & $3.53 \pm 0.12 *$ & $8.62 \pm 0.75 *$ & $0.94 \pm 0.07$ & $1.03 \pm 0.01$ \\
\hline & IV & $16.23 \pm 0.61$ & $5.51 \pm 0.31$ & $4.33 \pm 0.91$ & $0.61 \pm 0.09$ & $0.08 \pm 0.02$ \\
\hline & $\mathbf{V}$ & $16.95 \pm 0.54$ & $5.63 \pm 0.60$ & $4.17 \pm 1.01$ & $0.73 \pm 0.10$ & $0.07 \pm 0.01$ \\
\hline
\end{tabular}

Group I Normal control.

Group II Normal ration + HSCAS.

Group III T-2 toxin ration.

Group IV T-2 toxin ration physically treated.

Group V T-2 toxin ration chemically treated.

* Significantly different from normal control group, $\mathrm{p}<0.05$.

** Highly significantly different from normal control group, $\mathrm{p}<0.001$. 
Efficacy Of Physical And Chemical Treatment Of Broiler Ration To ...

Table (4): Serum biochemical parameters in different expermintal groups of Broiler chickens.

\begin{tabular}{|c|c|c|c|c|c|c|}
\hline $\begin{array}{l}\text { Time/ } \\
\text { week }\end{array}$ & Group & $\begin{array}{c}\text { ALT } \\
\text { (U/L.) }\end{array}$ & $\begin{array}{c}\text { AST } \\
\text { (U/L.) }\end{array}$ & $\begin{array}{l}\text { GGT } \\
\text { (U/L) }\end{array}$ & $\begin{array}{c}\text { BUN } \\
(\mathbf{m g} / \mathbf{d l})\end{array}$ & $\begin{array}{l}\text { Uric Acid } \\
(\mathrm{mg} / \mathrm{dl})\end{array}$ \\
\hline \multirow{5}{*}{$1 w$} & I & $16.97 \pm 1.19$ & $121.27 \pm 4.47$ & $8.73 \pm 1.6$ & $4.13 \pm 0.34$ & $3.56 \pm 0.81$ \\
\hline & II & $15.03 \pm 1.46$ & $118.83 \pm 3.73$ & $7.73 \pm 0.8$ & $4.03 \pm 0.10$ & $2.36 \pm 0.22$ \\
\hline & III & $16.63 \pm 0.98$ & $121.46 \pm 3.07$ & $8.40 \pm 0.4$ & $5.20 \pm 0.40$ & $2.03 \pm 0.61$ \\
\hline & IV & $15.75 \pm 0.87$ & $119.13 \pm 4.33$ & $7.97 \pm 1.3$ & $4.27 \pm 0.40$ & $2.56 \pm 0.79$ \\
\hline & $\mathbf{V}$ & $15.12 \pm 0.65$ & $122.13 \pm 0.30$ & $8.9 \pm 1.2$ & $4.87 \pm 0.50$ & $2.81 \pm 0.56$ \\
\hline \multirow{5}{*}{$2 \mathbf{w}$} & I & $27.3 \pm 1.04$ & $169.03 \pm 4.85$ & $9.97 \pm 0.97$ & $3.85 \pm 0.82$ & $3.28 \pm 0.40$ \\
\hline & II & $24.56 \pm 1.50$ & $165.25 \pm 3.90$ & $8.52 \pm 1.30$ & $3.93 \pm 0.60$ & $3.48 \pm 1.21$ \\
\hline & III & $34.28 \pm 1.07 *$ & $199.11 \pm 1.74 *$ & $9.41 \pm 0.60$ & $3.71 \pm 0.41$ & $3.12 \pm 0.24$ \\
\hline & IV & $31.58 \pm 1.60 *$ & $178.21 \pm 3.77 *$ & $9.80 \pm 0.81$ & $3.25 \pm 0.30$ & $3.18 \pm 0.27$ \\
\hline & V & $24.45 \pm 0.76$ & $165.65 \pm 3.45$ & $8.21 \pm 1.95$ & $3.13 \pm 0.91$ & $3.54 \pm 0.51$ \\
\hline \multirow{5}{*}{$3 w$} & I & $26.94 \pm 1.25$ & $159.50 \pm 5.9$ & $11.9 \pm 2.2$ & $2.73 \pm 0.09$ & $3.28 \pm 0.60$ \\
\hline & II & $26.93 \pm 0.54$ & $153 \pm 5.7$ & $12.4 \pm 1.2$ & $5.27 \pm 0.20$ & $3.46 \pm 1.10$ \\
\hline & III & $43.48 \pm 1.23^{* *}$ & $195.4 \pm 5.3 * *$ & $10.4 \pm 1.3$ & $7.30 \pm 1.60 * *$ & $5.22 \pm 1.05^{*}$ \\
\hline & IV & $35.23 \pm 1.6^{*}$ & $169.2 \pm 8.05^{*}$ & $12.6 \pm 1.1$ & $4.25 \pm 0.30$ & $2.26 \pm 0.50$ \\
\hline & $\mathbf{V}$ & $24.75 \pm 0.76$ & $153.6 \pm 5$ & $11.23 \pm 1.7$ & $4.18 \pm 0.51$ & $2.25 \pm 0.60$ \\
\hline \multirow{5}{*}{$4 w$} & I & $22.26 \pm 0.65$ & $165.50 \pm 5.12$ & $12.9 \pm 2.20$ & $4.71 \pm 1.21$ & $2.30 \pm 0.30$ \\
\hline & II & $23.33 \pm 1.05$ & $167.98 \pm 0.95$ & $13.50 \pm 2.10$ & $4.57 \pm 0.50$ & $3.22 \pm 0.50$ \\
\hline & III & $59.80 \pm 0.96^{* *}$ & $193.40 \pm 4.68 * *$ & $11.10 \pm 1.40$ & $8.82 \pm 1.61 *$ & $5.64 \pm 0.50 *$ \\
\hline & IV & $31.80 \pm 2.14^{*}$ & $173.33 \pm 4.33 *$ & $12.63 \pm 1.60$ & $8.44 \pm 0.50$ & $3.16 \pm 0.85$ \\
\hline & $\mathbf{V}$ & $21.60 \pm 1.58$ & $161.90 \pm 4.25$ & $11.73 \pm 1.50$ & $6.86 \pm 0.80$ & $2.72 \pm 0.50$ \\
\hline \multirow{5}{*}{$5 w$} & I & $23.73 \pm 1.12$ & $155.3 \pm 3.2$ & $13.13 \pm 2.2$ & $3.2 \pm 0.12$ & $2.94 \pm 0.20$ \\
\hline & II & $22.17 \pm 0.42$ & $155.1 \pm 2.95$ & $13.5 \pm 2.1$ & $3.68 \pm 0.8$ & $2.32 \pm 0.70$ \\
\hline & III & $47.83 \pm 0.26^{* *}$ & $188.2 \pm 3.7 * *$ & $12.9 \pm 1.4$ & $6.25 \pm 2.5^{*}$ & $6.45 \pm 1.20^{*}$ \\
\hline & IV & $30.60 \pm 1.72 *$ & $169.3 \pm 2.9 *$ & $11.9 \pm 2.5$ & $3.1 \pm 0.9$ & $3.56 \pm 0.61$ \\
\hline & $\mathbf{V}$ & $21.18 \pm 0.64$ & $159.0 \pm 1.68$ & $12.7 \pm 2.2$ & $3.73 \pm 0.34$ & $3.85 \pm 1.21$ \\
\hline \multirow{5}{*}{$6 \mathbf{w}$} & I & $23.53 \pm 0.48$ & $164.40 \pm 2.20$ & $14.0 \pm 1.5$ & $3.53 \pm 0.3$ & $2.0 \pm 0.3$ \\
\hline & II & $24.00 \pm 1.12$ & $167.60 \pm 40$ & $13.86 \pm 0.3$ & $2.1 \pm 1.09$ & $3.63 \pm 0.3$ \\
\hline & III & $41.00 \pm 1.13 * *$ & $199.40 \pm$ & $13.60 \pm 0.4$ & $6.63 \pm 1.2^{*}$ & $5.6 \pm 0.4^{*}$ \\
\hline & IV & $32.53 \pm 0.14^{*}$ & $176.00 \pm 0.30 *$ & $15.03 \pm 1.3$ & $2.35 \pm 0.5$ & $2.74 \pm 0.14$ \\
\hline & $\mathbf{V}$ & $25.13 \pm 0.60$ & $163.20 \pm 3.94$ & $14.73 \pm 1.4$ & $2.43 \pm 0.98$ & $3.0 \pm 0.4$ \\
\hline
\end{tabular}

Group I Normal control.

Group II Normal ration + HSCAS.

Group III T-2 toxin ration.

Group IV T-2 toxin ration physically treated.

Group V T-2 toxin ration chemically treated.

* Significantly different from normal control group, $\mathrm{p}<0.05$.

** Highly significantly different from normal control group, $\mathrm{p}<0.001$.

$\overline{\text { Kafrelsheikh Vet. Med. J. Vol. } 5 \text { No. } 1 \text { (2007) }}$ 
Table (5): Serum biochemical parameters in different experimental groups of Broiler chickens (continued).

\begin{tabular}{|c|c|c|c|c|c|c|c|}
\hline 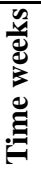 & $\stackrel{\varrho}{\overrightarrow{0}}$ & $\begin{array}{c}\text { Cholesterol } \\
\text { mg/dl. }\end{array}$ & $\begin{array}{l}\text { Glucose } \\
\text { mg/dl. }\end{array}$ & $\begin{array}{c}\text { Total protein } \\
\text { gm/dl. }\end{array}$ & $\begin{array}{c}\text { Albumin } \\
\text { gm/dl. }\end{array}$ & $\begin{array}{c}\text { Globulin } \\
\text { gm/dl. }\end{array}$ & A/G ratio. \\
\hline \multirow{5}{*}{1} & I & $117.36 \pm 2.15$ & $270.36 \pm 13.60$ & $3.23 \pm 0.20$ & $1.83 \pm 0.06$ & $1.17 \pm 0.18$ & $1.64 \pm 0.04$ \\
\hline & II & $118.96 \pm 5.12$ & $288.89 \pm 6.50$ & $3.23 \pm 0.80$ & $1.71 \pm 0.09$ & $1.13 \pm 0.19$ & $1.49 \pm 0.01$ \\
\hline & III & $118.83 \pm 1.5$ & $351.85 \pm 6.50 * *$ & $2.1 \pm 0.60^{*}$ & $1.18 \pm 0.11^{*}$ & $1.00 \pm 0.03$ & $1.17 \pm 0.02$ \\
\hline & IV & $120.3 \pm 3.40$ & $296.29 \pm 7.50$ & $3.13 \pm 0.09$ & $1.81 \pm 0.02$ & $1.21 \pm 0.07$ & $1.41 \pm 0.05$ \\
\hline & V & $110.4 \pm 6.50$ & $348.15 \pm 8.90$ & $3.3 \pm 0.15$ & $1.76 \pm 0.09$ & $1.32 \pm 0.06$ & $1.39 \pm 0.06$ \\
\hline \multirow{5}{*}{2} & I & $112.73 \pm 7.12$ & $288.89 \pm 6.50$ & $3.78 \pm 0.12$ & $3.08 \pm 0.06$ & $1.67 \pm 0.07$ & $1.93 \pm 0.01$ \\
\hline & II & $114.3 \pm 2.10$ & $322.22 \pm 3.30$ & $4.05 \pm 0.50$ & $3.15 \pm 0.06$ & $1.85 \pm 0.33$ & $1.76 \pm 0.05$ \\
\hline & III & $110.83 \pm 5.69$ & $333.33 \pm 6.50 * *$ & $2.53 \pm 0.03 *$ & $1.58 \pm 0.02 *$ & $1.02 \pm 0.34 *$ & $1.54 \pm 0.01$ \\
\hline & IV & $116.06 \pm 7.14$ & $307.4 \pm 2.98$ & $4.06 \pm 0.08$ & $3.49 \pm 0.06$ & $1.48 \pm 0.06$ & $1.80 \pm 0.03$ \\
\hline & $\mathrm{V}$ & $115.1 \pm 4.40$ & $288.89 \pm 9.03$ & $4.94 \pm 0.10$ & $3.25 \pm 0.12$ & $1.85 \pm 0.06$ & $1.96 \pm 0.03$ \\
\hline \multirow{5}{*}{3} & I & $124.2 \pm 1.80$ & $281.48 \pm 16.40$ & $3.85 \pm 0.05$ & $2.33 \pm 0.03$ & $1.40 \pm 0.11$ & $1.66 \pm 0.06$ \\
\hline & II & $122.76 \pm 8.70$ & $242.74 \pm 9.98$ & $3.78 \pm 0.07$ & $2.38 \pm 0.05$ & $1.45 \pm 0.02$ & $1.72 \pm 0.01$ \\
\hline & III & $105.23 \pm 2.30 * *$ & $181.5 \pm 5.10 * *$ & $2.9 \pm 0.15^{*}$ & $1.56 \pm 0.15 *$ & $1.18 \pm 0.02 *$ & $1.13 \pm 0.04$ \\
\hline & IV & $127.96 \pm 2.30$ & $270.37 \pm 7.60$ & $3.87 \pm 0.03$ & $2.4 \pm 0.11$ & $1.4 \pm 0.03$ & $1.65 \pm 0.05$ \\
\hline & $\mathrm{V}$ & $123.03 \pm 1.50$ & $233.3 \pm 6.50$ & $3.75 \pm 0.09$ & $2.2 \pm 0.09$ & $1.55 \pm 0.10$ & $1.67 \pm 0.04$ \\
\hline \multirow{5}{*}{4} & I & $122 \pm 1.97$ & $288.15 \pm 6.50$ & $3.55 \pm 0.16$ & $2.26 \pm 0.12$ & $1.5 \pm 0.10$ & $1.63 \pm 0.12$ \\
\hline & II & $121.4 \pm 7.10$ & $278.15 \pm 6.40$ & $3.82 \pm 0.09$ & $2.64 \pm 0.10$ & $1.1 \pm 0.04$ & $1.76 \pm 0.03$ \\
\hline & III & $109.86 \pm 6.90$ & $221.48 \pm 2.90 *$ & $2.2 \pm 0.16^{*}$ & $1.23 \pm 0.20 *$ & $1.03 \pm 0.40$ & $1.06 \pm 0.06^{* *}$ \\
\hline & IV & $121.8 \pm 4.30$ & $287.03 \pm 3.77$ & $3.84 \pm 0.26$ & $2.48 \pm 0.12$ & $1.25 \pm 0.13$ & $1.79 \pm 0.08$ \\
\hline & V & $122.5 \pm 6.80$ & $285.18 \pm 2.10$ & $3.43 \pm 0.18$ & $2.33 \pm 0.11$ & $1.2 \pm 0.40$ & $1.51 \pm 0.11^{* *}$ \\
\hline \multirow{5}{*}{5} & I & $126.2 \pm 6.02$ & $271.85 \pm 2.50$ & $4.25 \pm 0.14$ & $2.86 \pm 0.09$ & $1.63 \pm 0.15$ & $1.74 \pm 0.03$ \\
\hline & II & $125.43 \pm 7.50$ & $272.3 \pm 4.04$ & $3.82 \pm 0.30$ & $2.56 \pm 0.20$ & $1.48 \pm 0.34$ & $1.88 \pm 0.10$ \\
\hline & III & $89.9 \pm 3.90 * *$ & $237.13 \pm 8.40^{*}$ & $3.1 \pm 0.01 *$ & $1.84 \pm 0.04 *$ & $1.01 \pm 0.16$ & $0.96 \pm 0.20$ \\
\hline & IV & $122.8 \pm 2.50$ & $272.8 \pm 3.30$ & $4.24 \pm 0.40$ & $2.66 \pm 0.08$ & $1.18 \pm 0.02$ & $1.81 \pm 0.03$ \\
\hline & V & $120.95 \pm 4.90$ & $269.9 \pm 2.80$ & $4.14 \pm 0.30$ & $2.8 \pm 0.07$ & $1.26 \pm 0.20$ & $1.86 \pm 0.03$ \\
\hline \multirow{5}{*}{6} & I & $130.8 \pm 1.50$ & $261.9 \pm 9.70$ & $3.3 \pm 0.12$ & $2.13 \pm 0.09$ & $1.13 \pm 0.09$ & $1.71 \pm 0.04$ \\
\hline & II & $131.4 \pm 2.10$ & $269.3 \pm 2.90$ & $4.06 \pm 0.32$ & $2.91 \pm 0.15$ & $1.43 \pm 0.09$ & $1.65 \pm 0.04$ \\
\hline & III & $111.86 \pm 2.90 * *$ & $216.1 \pm 2.20 *$ & $2.76 \pm 0.09 *$ & $1.57 \pm 0.03 *$ & $1.21 \pm 0.06 *$ & $1.30 \pm 0.06$ \\
\hline & IV & $134.125 \pm 1.70$ & $263.4 \pm 3.40$ & $3.58 \pm 0.09$ & $2.8 \pm 0.04$ & $1.70 \pm 0.05$ & $1.61 \pm 0.01$ \\
\hline & V & $133.7 \pm 4.80$ & $265.5 \pm 2.80$ & $3.36 \pm 0.12$ & $2.65 \pm 0.05$ & $1.73 \pm 0.12$ & $1.70 \pm 0.20$ \\
\hline
\end{tabular}

Group I Normal control.

Group III T-2 toxin ration.
Group II Normal ration + HSCAS.

Group IV T-2 toxin ration physically treated.

Group V T-2 toxin ration chemically treated.

* Significantly different from normal control group, $\mathrm{p}<0.05$.

** Highly significantly different from normal control group, $\mathrm{p}<0.001$.

Table (6): T-2 toxin residues in chicken meat of experimentally T-2 toxinated chickens.

\begin{tabular}{||c||c|}
\hline \multicolumn{1}{|c|}{ Group } & Concentration of T-2 toxin $(\boldsymbol{\mu g} / \mathbf{k g})$. \\
\hline \hline III T-2 toxin ration. & $900.0 \pm 2.00$ \\
IV T-2 toxin ration physically treated. & $600.0 \pm 3.10$ \\
V T-2 toxin ration chemically treated. & $41.0 \pm 1.12$ \\
\hline
\end{tabular}

* Control group was free of T-2 toxin residues.

Kafrelsheikh Vet. Med. J. Vol. 5 No. 1 (2007) 


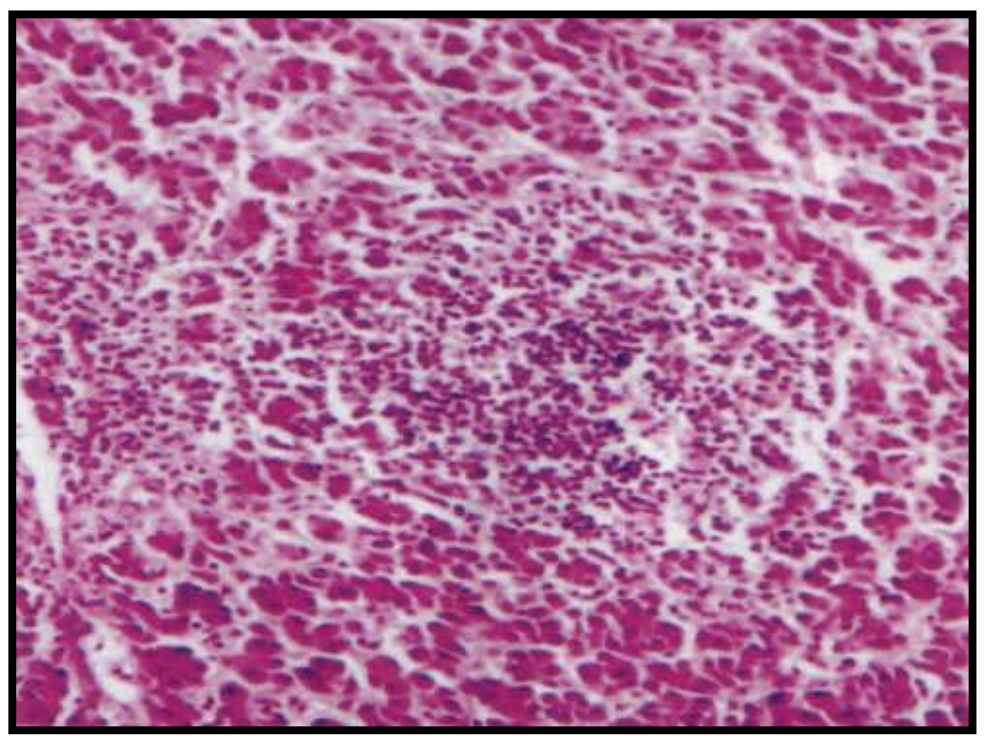

Fig. (1): Liver of chicken exposed to T-2 toxin 4 weeks post treatment showed focal leukocytes infiltration. (H\&E., X100)

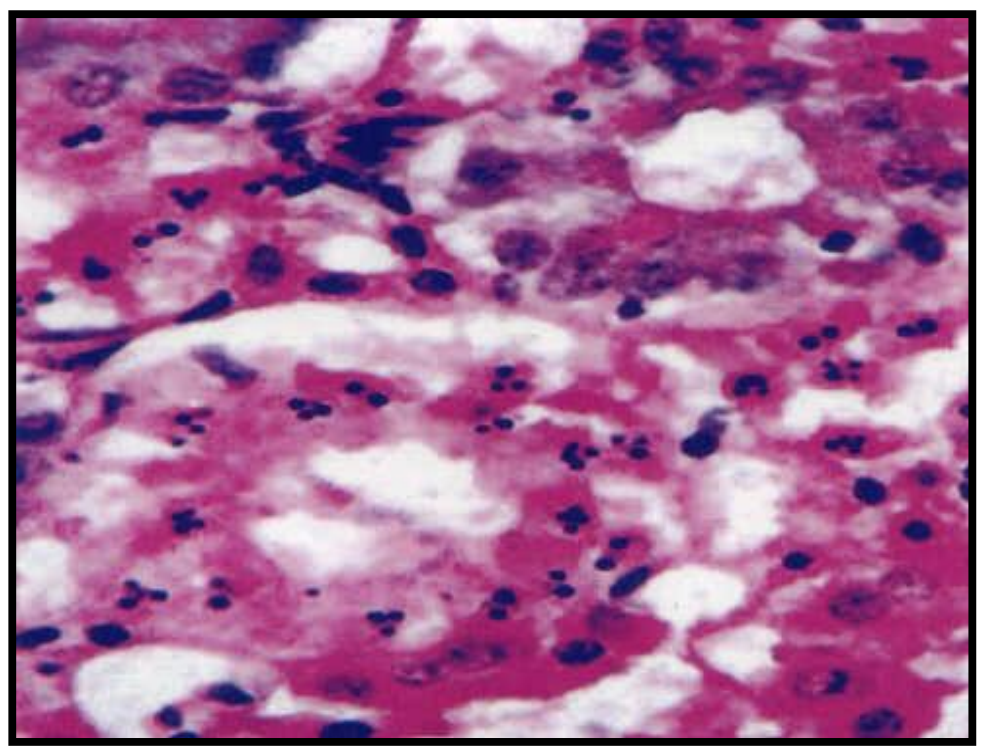

Fig. (2): Kidney of chicken exposed to T-2 toxin 3 weeks post treatment showed marked apoptotic changes. (H\&E., X100) 


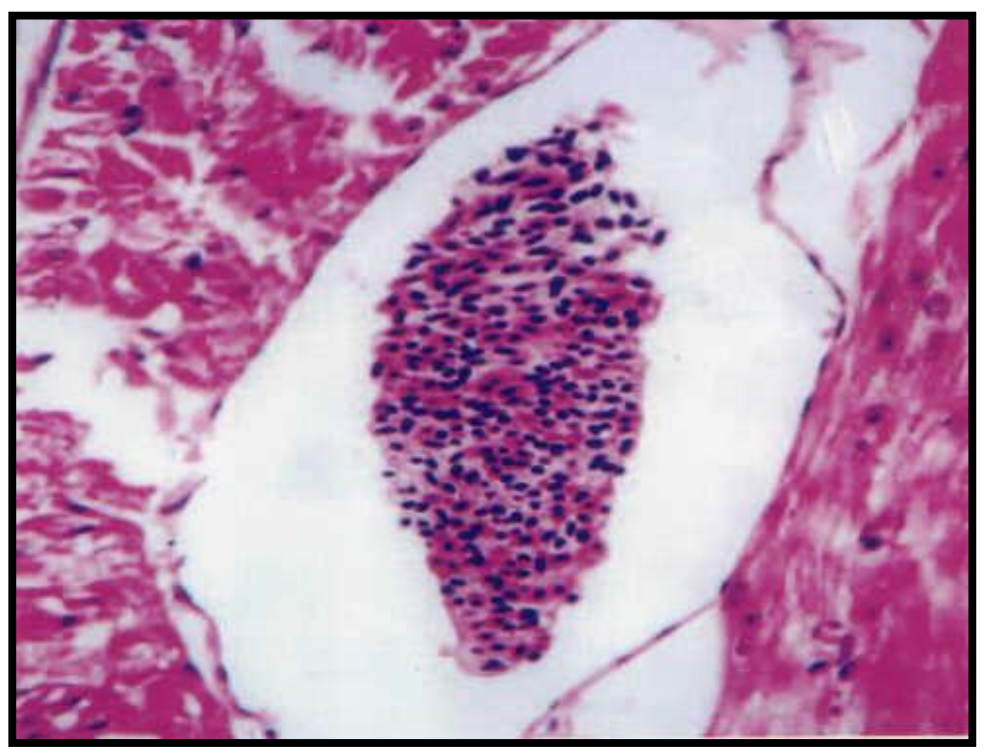

Fig. (3): Heart of chicken exposed to T-2 toxin 4 weeks post treatment showed congestion and leucocytic infiltration. (H\&E., X200)

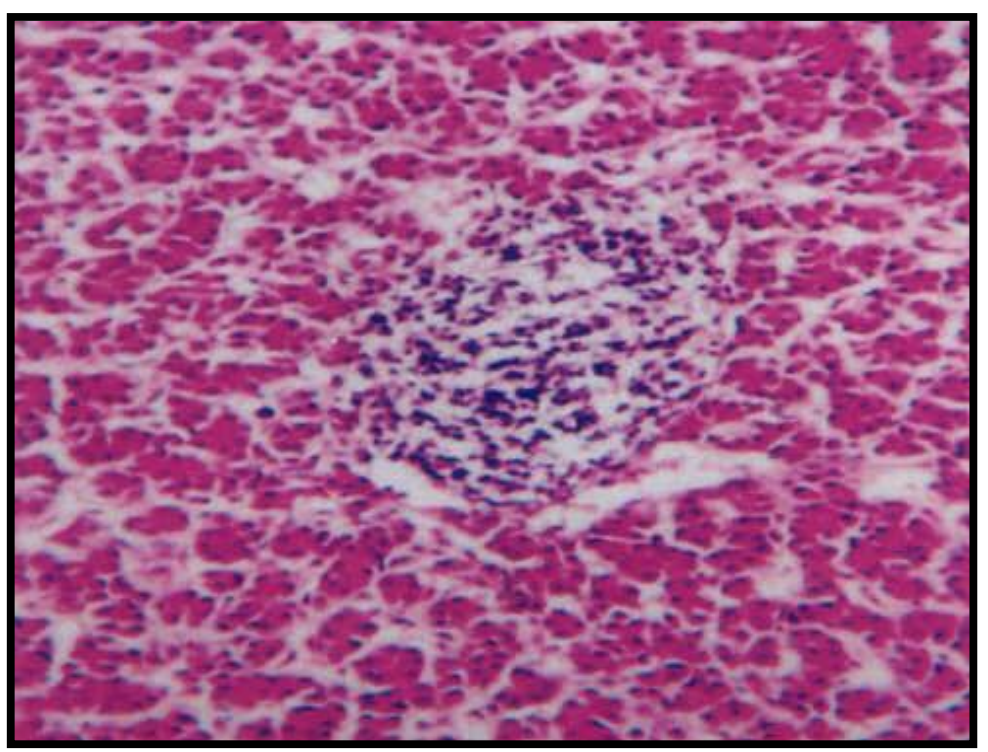

Fig. (4): Liver of chicken exposed to T-2 toxin ration 3 weeks post treatment treated physically showed focal leucocytic infiltration.(H\&E.,X 100) $\overline{\text { Kafrelsheikh Vet. Med. J. Vol. } 5 \text { No. } 1 \text { (2007) }}$ 


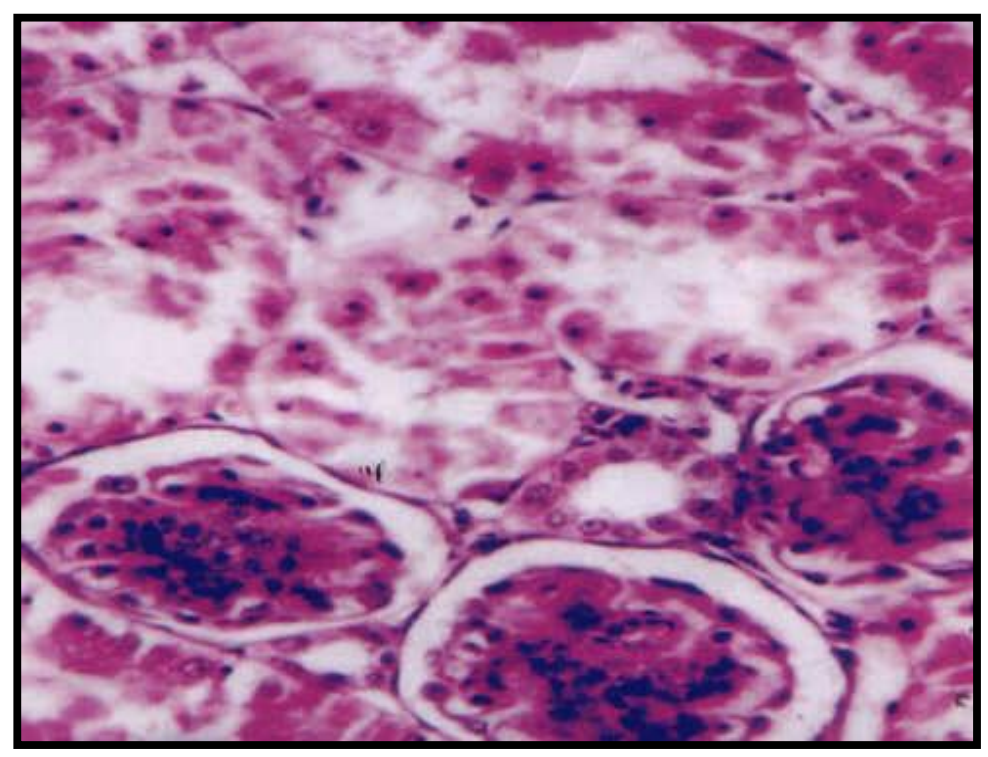

Fig. (5): Kidney of chicken exposed to T-2 toxin ration treated physically 6 weeks post treatment showed nuclear condensation. (H\&E., X 400)

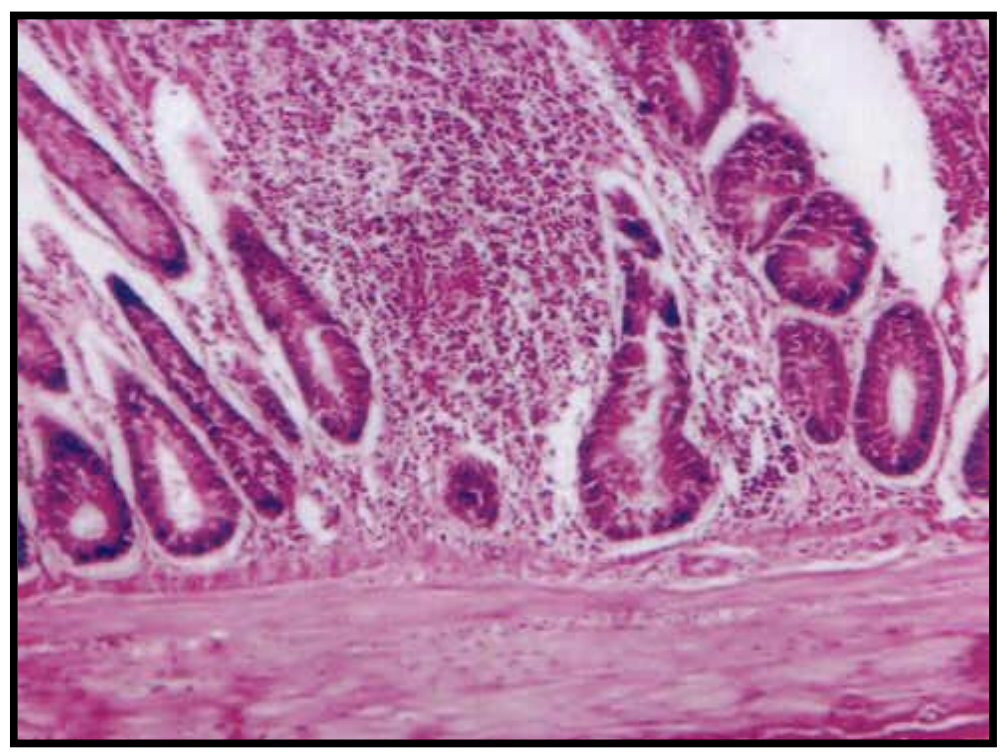

Fig. (6): Intestin of chicken exposed to T-2 toxin ration treated physically 5 weeks post treatment showed mild interitis. (H\&E., X 40) 


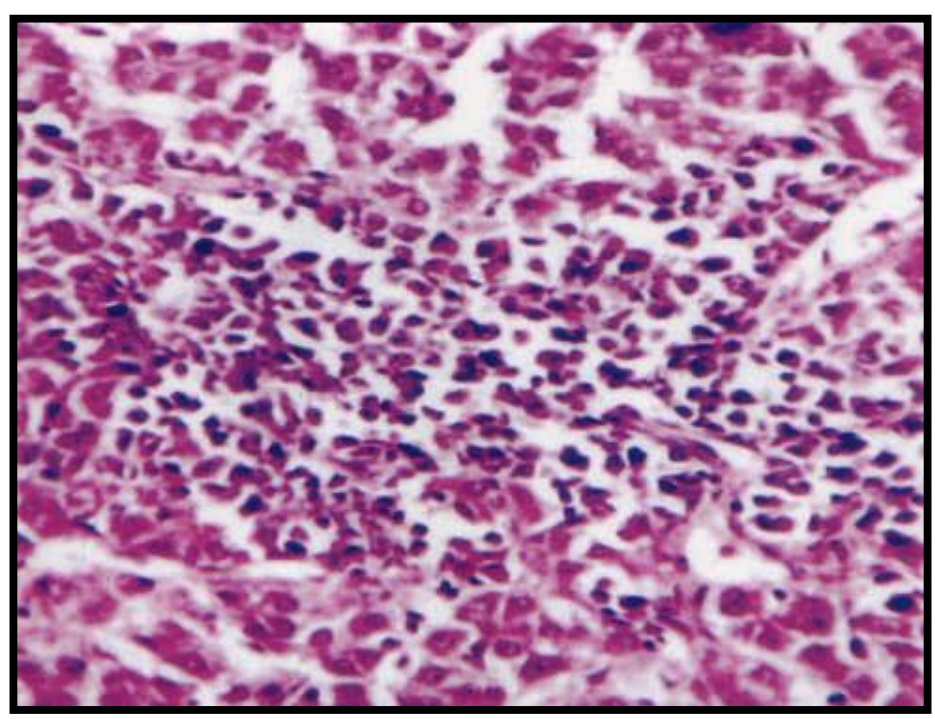

Fig. (7): Liver of chicken exposed to T-2 toxin ration treated chemically 4 weeks post treatment showed focal leucocytic infiltration. (H\&E., X 400)

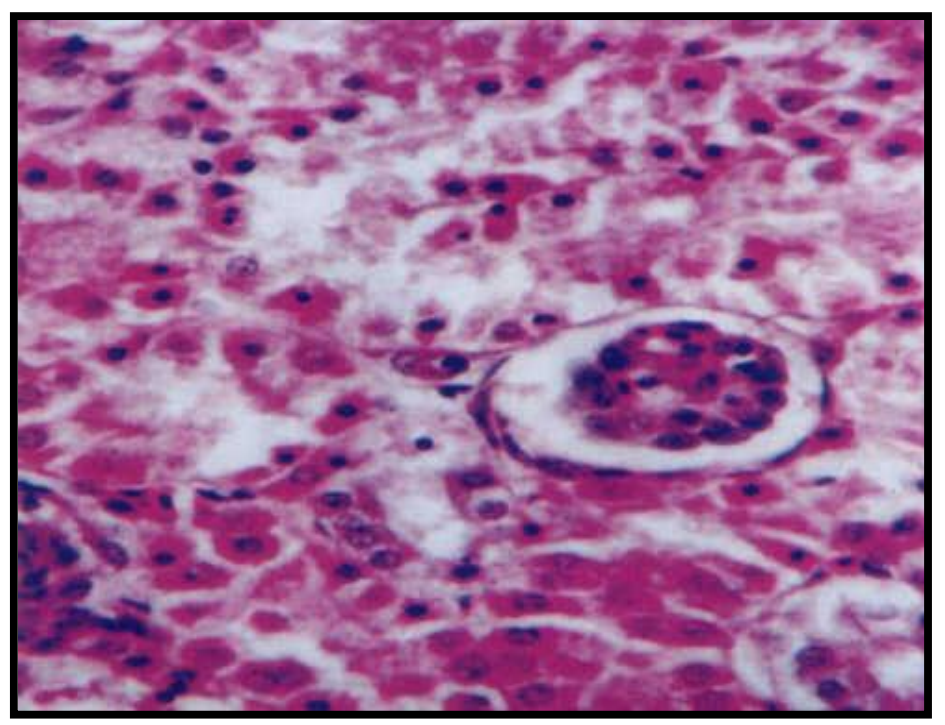

Fig. (8): Kidney of chicken exposed to T-2 toxinration treated chemically 6 weeks post treatment showed nuclear condensation. (H\&E., X 40) 


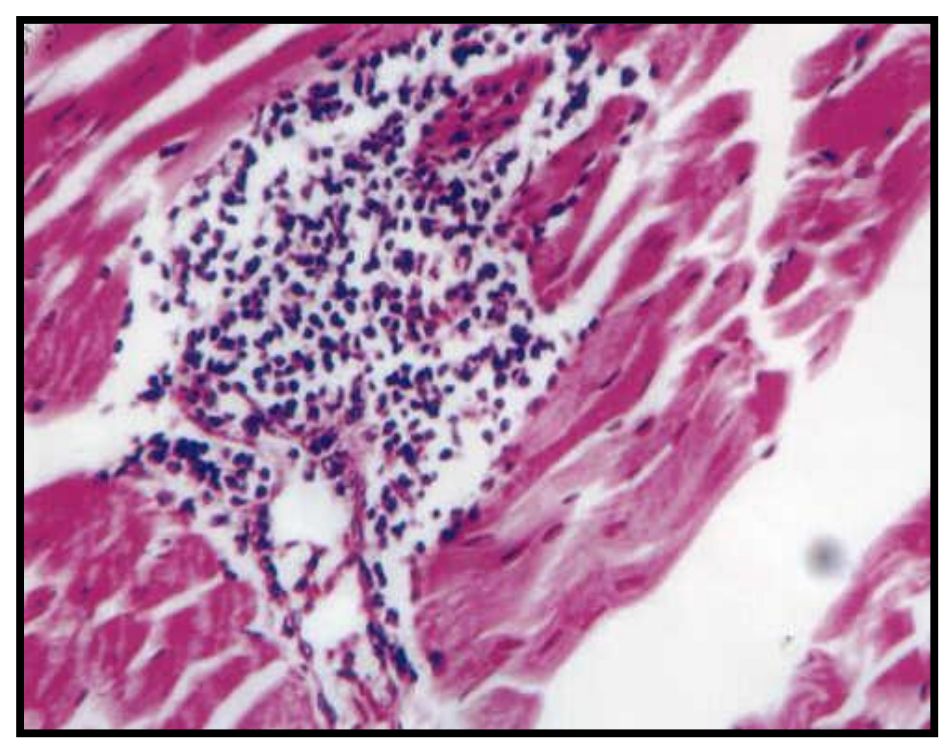

Fig. (9): Heart of chicken exposed to T-2 toxin ration treated chemically 5 weeks post treatment showed focal leucocytic infiltration. (H\&E., X 400)

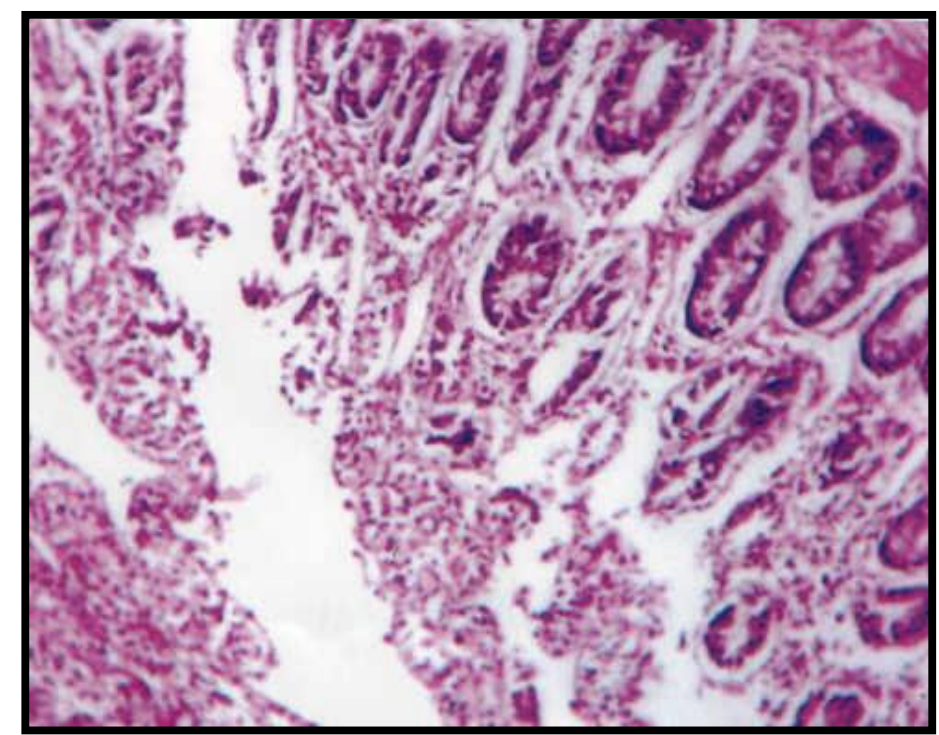

Fig. (10): Intestine of chicken exposed to T-2 toxin ration treated Chemically 4weeks post treatment showed mild interitis. (H\&E., X 40) 


\section{DISCUSSION}

Some common soil fungi may contaminate grains during transportation and storage, and produce mycotoxines that are consumed by animals and poultry. Fusarium spp. Produce a mycotoxin known as T-2 toxin which causes reduced performance and severe oral lesions in poultry (Kubena et al., 1995a). Extensive research has been conducted to counter the occurence of mycotoxicosis by physical, chemical, nutritional or biological approaches.

Clinical signs of severe depression, ruffled feathers and reduction in feed consumption and body weight were obviously observed in all toxinated chicken groups in this experiment. A similar signs were recorded by Wyatt et al., (1975a); Mishra et al., (1987); Kubena et al., (1997) and Casarin et al., (2006). The decrease of body weight is most likely associated with the direct effect of the toxin on protein synthesis as T-2 toxin acts as protein synthesis inhibitor (Cundliffe et al., 1974 ; McLaughlin et al., 1977 and Kubena et al., 1994).

Erythrogram showed significant decrease in $\mathrm{Hb}, \mathrm{PCV}$ and RBCs count in the 3rd group (received T-2 toxin contaminated ration) and the 5th group (received T-2 toxin ration treated chemically) from the 3rd week till the end of the experiment. Similar results were reported by $\boldsymbol{C h i}$ et al., (1981); Huff et al., (1988); Raju and Devegowda (2000); Kamalavenkatesh (2003); Parent-Massin, (2004) and Krishnamoorthy et al., (2006). These changes may be due to the direct effect of the toxin on the hematopoietic tissues (Wyatt et al., 1975 b; Hoerr et al., 1982 and Parent- Massin, 2004). Furthermore, T-2 toxin could cause membrane changes resulting in dramatic alteration in the red cell morphology leading to anemia (Gongyossy-Issa et al., 1986). Another possible

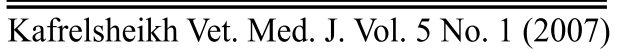


explanation for the reduction of $\mathrm{Hb}$ value is due to iron and essential minerals' deficiency which are required for $\mathrm{Hb}$ synthesis as copper, cobalt and molybdenum as a result of impairment of the absorptive capacity of the intestine (Greenway and Puls, 1976 and Hoerr et al., 1981). Leucogram showed significant leucopenia accompanied with heteropenia and lymphocytosis in the 3 rd group of chicken from the $2^{\text {nd }}$ week till the end of the experiment. These changes agree with Wyatt et al., (1975 b) and Chi et al. (1981) and may be as a result of the toxic effect of T-2 toxin on the hematopoietic tissues as reported by Wyatt et al. (1975 b); Hoerr et al. (1982) and Parent-Massin (2004). Histopathological examination revealed presence of multifocal leucocytic infiltration, mainly of mononuclear within the hepatic parenchyma which coincide with the observed pattern of leucocytes.

Serum biochemistry analysis showed significant increase in the activities of aminotransferases in the $3^{\text {rd }}$ group which exposed to T-2 toxin and the $4^{\text {th }}$ group that received $\mathrm{T}-2$ toxin ration treated physically by heat from the $2^{\text {nd }}$ week till the end of the experiment. These findings concurred with reports of Raju and Devegowda(2000); Kamalavenkatesh, (2003) and Krishnamoorthy et al. (2006) and may be resulted from liver damage produced by the toxin (Fudge, 2000 and Harris, 2000). Levels of serum BUN and uric acid revealed significant elevation from the $3^{\text {rd }}$ week till the end of the experiment. Similar findings were reported by Chi et al. (1977a) and Grizzle et al. (2004). Such increase could be due to development of kidney lesions during excretion of the toxin and its metabolities through the kidney with urine (Ragheb, 1994 and Harris, 2000) which was approved histopathologically. Level of glucose showed significant increase in the first two weeks then showed significant decrease from the third week till the end of the experiment. The observed changes could be attributed to inefficient utilizatioin of 
glucose due to deficiency of insulin (during the early period of the experiment and to hepatic damage latter on in the toxin fed birds. Rather similar results were reported by Raina et al.(1991)and kamalavenkatesh (2003). Cholesterol values showed significant decrease from the $1^{\text {st }}$ week till the end of the experiment in the T-2 toxin group. This result concurred with reports of (Chi et al., 1977a; Edrington et al., 1997; Bailey et al., 1998; Kamalavenkatesh, 2003 and Krishnamoorthy et al., 2006). The hypocholesterolaemia is most likely attributed to inhibition of cholesterol biosynthesis due to liver involvement (Kubena et al., 1989). Significant reduction in total protein, albumin and globulin values were observed by the end of the first week till the end of the experiment. These findings agree with(Boonchuvit et al.,1975;Huff et al., 1988;Kamalavenkatesh, 2003 and Krishnamoorthy et al., 2006). Hypoalbuminemia and hypoglobulinemia observed may be attributed to the effect of T-2 toxin on protein synthesis (Cundliffe et al., 1974 and McLaughlin et al., 1977) or to the reduction in feed consumption, and hepatic damage as the liver is the major organ of protein synthesis specially albumin (Kaneko et al., 1997 and Krishnamoorthy et al., 2006).

Gross pathological examination of the 3rd group (T-2 toxinated group) revealed hemorrhagic lesions in kidneys, intestine and heart. Such findings agree with recordes of Shlosberg et al (1984). Heart dilatation also was observed which could be due to the severe anemia observed (Kjeldsberg et al.,1991).Histopathological examination revealed necrotic changes of the renal tubules, entritis and necrosis of the lining epithelial cells, and hepatocellular degeneration in birds received T-2 toxin. Such results are in accordance to those reported by Ványi et al.(1994); Rachid et al. (2000); kamalavenkatesh et al. (2003) and Grizzle et al. (2004).

Measurment of T-2 toxin residues revealed presence of the toxin in the chicken muscles that agree with the reports of (Chi et al., 1978 and WHO, 1990). 
In conclusion: physical treatment of the ration by using of dry heat at $200^{\circ} \mathrm{C}$ for one hour decreases the toxic effects of $\mathrm{T}-2$ toxin as the treatment improved the hemogram and the toxic effect on the kidneys and to a lesser degree on the liver. Ragheb (1994) reported that physical treatment by using of dry heat causes destruction of almost $50 \%$ of the toxin. Treatment was not valiable in improving the body weight gain of birds and the toxin residues in the chicken meat. Chemical treatment by using of HSCAS causes adsorption to the T-2 toxin thus minimising its toxic effects (Casarin et al., 2006). The present study showed that using of chemical treatment was effective as the body weight gain, hemogram, liver and kidney functions were within normal. Moreover the histopathological picture was milder than in the $3^{\text {rd }}$ group, and there was a great reduction of the toxin residues that constitute a puplic health hazard. The present results are in accordance with that reported by Huff et al. (1992).

\section{REFERENCES}

- Bailey, R.H.; Kubena, L.F.; Harvey, R.B.; Buckley S.A. and Rottinghaus, G.E. (1998): Efficacy of various inorganic sorbents to reduce the toxicity of Aflatoxin and T-2 toxin in broiler chickens. Poult. Sci., 77: 1623-1630.

- Bancroft, J.D. and Stevens, A. (1996): Theory and Practice of Histological Techniques. Churchill liveingstone, New York.

- Beasley, V.R. (1989): Trichothecene mycotoxicosis: Pathophysiologic effects. Vol. I. CRC Press, Boca Raton, Fla.

- Beng, C.G. and Lim, K.L. (1973): Determination of serum albumin. Am. J. Clin. Pathol., 59: 14-19. 
- Boonchuvit, B.; Hamilton, P.B. and Burmeister, H.R. (1975): Interaction of T-2 toxin with salmonella infections of chickens. Poult. Sci., 54:1693-1696.

- Casarin, A.; Forat, M.; Soto, E. and Zaviezo, D. (2006): Evaluation of the efficacy a commercial purified phylosilicate to reduce the toxicity of T-2 toxin in broiler chicks. Int. Poult. Sci. Forum. Georgia World Congress Center, Atlanta, Georgia.

- Chi, M.S.; Mirocha, C.J.; Kurtz, H.J.; Weaver, G.; Bates, F. and Shimoda, W. and Burmeister, H.R. (1977a): Acute toxicity of T-2 toxin in broiler chicks and laying hens. Poult. Sci., 56: 103-116.

- Chi, M.S.; Robison, T.S.; Mirocha, C.J.; Swanson, S.P. and Shimoda, W. (1978): Excretion and tissue distribution of radioactivity from tritium labeled T-2 toxin in chicks.Toxicol. Appl. Pharmacol., 45: 391-402.

- Chi, M.S.; El-Halawani, M.E.; Waibel, P.E. and Mirocha, C.J. (1981): Effect of T-2 toxin on brain catecholamines and selected blood components in growing chickens. Poul. Sci., 60 (1): 137-141.

- Corrier, D.E.(1991): Mycotoxicosis. Mechanisms of immunosupression. Vet. Immunol. Immunopathol., 30: 73-87.

- Council for Agricultural Science and Technology, (1988): pages 1-91 in: Mycotoxin: Economic and Health Risk. K.A. Nisi, ed. Council for Agricultural Science and Technology, Ames, IA. 
- Cundliffe, E., Cannon, M. and Davies, J. (1974): Mechanism of inhibition of eukaryotic protein synthesis by trichothecenes fungal toxins.Proc. Nat. Acad. Sci., 77 (1): 30-34.

- Edrington, T.S.; Kubena, L.F.; Harvey, R.B. and Rottinghaus, G.E. (1997): Influence of superactivated charcoal on the toxic effects of aflatoxin or T-2 toxin in growing broilers. Poult. Sci., 76 (9): 1205-1211.

- El-Hamaky, A.M.A. (2001): Studies on toxigenic Fusarium species in feeds. M.V.Sc. Thesis, Faculty of Vet. Med., Cairo Univ., Egypt. M.V.Sc. Thesis, Faculty of Vet. Med., Cairo Univ., Egypt.

- Fudge, A.M. (2000): Avian liver and gastrointestinal testing. In: Laboratory medicine: avian and exotic pets. Fudge, A.M. Ed. Saunders, W.B. Co., Philadelphia, PA., 47-55.

- Gongyossy-Issa,M.I.C.; Khanna,V.and Khachatourians,G.G. (1986): Changes induced by $\mathrm{T}-2$ toxin in the erythrocytes. Food Chem. Toxicol., 24: 311-317.

- Greenway, J.A. and Puls, R. (1976): Fusariotoxicosis from barley in British Columbia. I. Natural occurrence and diagnosis. Can. J. Comp. Med., 40: 12-15.

- Grizzle, J.M.; Kersten, D.B.; Mc Cracken, M.D.; Houston, A.E. and Saxton. A.M. (2004): Determination of the acute 50 lethal dose of T-2 toxin in adult bobwhite quail: additional studies on the effect of T-2 mycotoxin on blood chemistry and the morphology of internal organs. Av. Dis., 48 (2): 392-399. 
- Grizzle, J.M.; Kersten, D.B.; Houston,A.E. and Saxton, A.M. (2005): Effect of the chronic V.S. Intermittent exposure of T-2 toxin on reproductive performance in Bobwhite quail. Int. J. of Poult. Sci., 4 (2) 71-75. Jan.-Feb.: 2-3.

- Harris, D.J. (2000): Clinical tests. In: Avian medicine. Tully, T.N.; Lawton, M.P.C. and Dorrestein, G.M., Eds. Butterworth and Heinemann, Oxford, England, 43-51.

- Hayes, M.A.; Bellamy, J.E.C. and Schiefer, H.B. (1980): Subacute toxicity of dietary T-2 toxin in mice: Morphological and haematological effects.

- Henry, R.J.; Cannon, D.C. and Winkelman, J.W. (1974): Determin-ation of cholesterol and total protein. Clinical Chemistry Principles and Technics. Harper and Row, New York, 1440. Vet. Rec., 74: 156-167.

- Hoerr, F.J.; Carlton, W.W. and Yagen, B. (1981): Mycotoxicosis caused by a single dose of $\mathrm{T}-2$ toxin or diacetoxyscirpenol in broiler chickens. Vet. Path., 18 (5): 652664.

- Hoerr, F.J.; Carlton, W.W.; Yagen, B. and Joffe, A.Z. (1982): Mycotoxicosis produced in broiler chickens by multiple doses of either T-2 toxin or diaceatoxyscirpenol. Av. Path., 11 (3): 369-383.

- Huff, W.E.; Harvey, R.B.; Kubena, L.F. and Rottinghaus, G.E. (1988): Toxic synergism between aflatoxin and T-2 toxin in broiler chickens. Poul. Sci., 67 (10): 1418-1423.

- Huff, W.E.; Kubena, L.F.; Harvey, R.B and Phillips,T.D. 
(1992): Efficacy of hydrated sodium calcium aluminosilicate to reduce the individual and combined toxicity of aflatoxin and ochratoxin A. Champaign,Ill.: Poultry Science Association ; Poultry science, Jan. vol. 71(1): 64-69.

- Jain, N.C. (1986): Schalm's Veterinary Hematology. $4^{\text {th }}$ Ed., Lea and Fenbiger, Philadelphia, PA.

- Janovitz, E.B. (1988): Embryotoxicity of Fusarium mycotoxins, zearalenone and $\mathrm{T}-2$ toxin, in rats. Dissertation Abstracts Internati-onal, 48 (10): 2833.

- Joffe, A. Z. (1971): Alimentary toxic aleukia (ATA). In Microbial. Toxin., Vol.7. S.Kadies,A.Ceigler and S.J.AJL(Eds.), Academic Press, New York.

- Kamalavenkatesh, P.(2003): Individual and combined effects of cyclopiazonic acid and T-2 toxin in broiler chicken. M.V.Sc. thesis submitted to Tamilnadu Vet.and Anim Sci. Univ., Chennai 600 0051, India.

- Kaneko, J.J.; Harvey, J.W. and Bruss, M.L. (1997): Clinical bioche-mistry of domestic animals, $5^{\text {th }}$ Edn. Academic Press, California.

- Kjeldsberg,C.;Butter,E.;Bell,C.;Hougie,C.; Foucar, K. and Savage, R. (1991): Practical Diagnosis of Haematological Disorders.Revised ed. American Society of Clinical Pathologists. Chicago.

- Krishnamoorthy, P.; Vairamuthu, S.; Balachandran, C. and Mur-alimanohar, B.(2006): Chlorpyriphos and T-2 toxin induced haemato-biochemical alterations in broiler chicken. Int. J. of Poult. Sci., 5 (2): 173-177. 
- Kubena, L.F.; Huff, W.E.; Harvey, R.B.; Phillips, T.D. and Rottinghaus, G.E. (1989): Individual and combined toxicity of deoxynivalenol and T-2 toxin in broiler chickens. Poult. Sci., 68 (5): 622-626.

- Kubena, L.F.; Smith, E.E.; Gentles, A.; Harvey, R.B.; Edinbergton, T.E.; Phillips, T.D. and Rottinghaus, G.E. (1994): Individual and combined toxicity of T-2 toxin and cyclopiazonic acid in broiler chicks. Poult. Sci., 73: 1390-1397.

- Kubena, L.F.; Edrington, T.S.; Kamps-Holtzapple, C.; Harvey, R.B.; Elissalde, M.H. and Rottinghaus, G.E. (1995a): Influence of Fumonisin $\mathrm{B}_{1}$, present in Fusarium moniliforme culture material and T-2 toxin on turkey poults. Poult. Sci., 74 (2): 306-313.

- Kubena,L.F.;Edrington,T.S.; Harvey, R.B.; Buckley, S.A.; Phillips, T.D.; Rottinghaus, G.E. and Caspers, H.H. (1997): Individual and combined effects of Fumonisin $B_{1}$ present in Fusarium moniliforme culture Material and T-2 toxin or Deoxynivalenol in broiler chicks. Poult. Sci., 76: 1239-1247.

- McLaughlin, C.S.; Vaughan, M.H.; Campbell, I.M.; Stafford, C.M. and Hansen, B.S. (1977): Inhibition of protein synthesis by trichothecenes. In Rodricks, J.V.; Hesseltine, C.W. and Mehleman, M.A. (eds) Mycotoxins in Human and Animal Health, pp. 263-273.

- Mishra, U.K.; Dwarkanath, P.K. and Hossain, M.I. (1987): Clinical manifestation and hepatic trace minerals in growing 
chickens as influenced by T-2 toxin. Ind. J. of Anim. Sci., 57 (10): 1069-1074.

- Parent-Massin,D.(2004):Haematotoxicity of trichothecenes. Toxicol. Lett. 153 (1): 75-81.

- Rachid, M.A.; Vasconcelos, A.C. and Nunes, V.A. (2000): Apoptosis in the lymphoid depletion induced by T-2 toxin in broiler chicks. Histomorphometry of the bursa of Fabricius. Arquivo Brasilerio de Medicina Vetrinária ezootecnia, 52 (6): 592-598.

- Ragheb, R.R. (1994): Studies on fusariotoxicosis in farm animals. Ph.D. Thesis, Faculty of Vet. Med., Cairo Univ., Egypt.

- Raina, J.S; Roy, K.S. and Singh, B. (1991): Biochemical and histochemical studies in experimental mycotoxicasis in chicks. Ind. J. Anim. Sci., 62: 1276-1281.

- Raju, M.V.L.N. and Devegowda, G. (2000): Influence of estrified-glucomannan on performance and organ morphology, serum biochemistry and haematology in broilers exposed to individual and combined mycotoxicosis (aflatoxin, ochratoxin andT-2 toxin). Br. Poult. Sci., 41: 640-650.

- Real, R.N. and Croft, D.J. (1961): Colorimetric determination of blood urea nitrogen. Clin. Path., 14: 418.

- Reitman,S. and Frankel,S.(1957): A colorimetric method for 
the determination of serum glutamic oxaloacetic and glutamic pyruvic transmaminases. Am.J.Clinc.Path. , 28:56-63.

- Richard (2003): Mycotoxins: Risk in plant, Animal and Human Systems.- Ames.

- Shlosberg, A.; Weisman, Y.; Handji, U.; Yagen, B. and Shore, L. (1984): A sever reduction in egg laying in a flock of hens associated with trichothecenes mycotoxins in the feed. Veterinary and human toxicology, 26: 384-386.

- Snedecor, W.G. and Cochran, W.G. (1967): Statistical Methods. $6^{\text {th }}$ Ed. Iowa Univ. Press, Ames, Iowa, U.S.A.

- Trinder, P. (1969): Enzymatic determination of glucose. Ann. Clin. Biochem.

- Ványi, A.; Bata, A. and Kovács, F. (1994): Effect of T-2 toxin on the egg yield and hatchability in geese. Acta Vet. Hung., 42 (1): 79-85.

- Visconti, A. and Mirocha, C.J. (1985): Identification of various T-2 toxin metabolites in chicken excreta and tissues. Appl. And Enviro. Microb.,49 (5) 1246-1250

- Whitfield, J.B.; Moss, D.W.; Neale, G.; Orme, M. and Breckenridge, A. (1973): Br. Med. J., 1: 136.

- WHO, Geneva (1990): International programme on chemical safety., Environmental Health Criteria 105, Selected Mycotoxins: Ochratoxins, Trichothecenes, Ergot. 
- Wyatt, R.D.; Hamilton, P.B. and Burmeister, H.R. (1975a): Altered feathering of chicks caused by T-2 toxin. Poult. Sci., 54: 1042-1045.

- Wyatt, R.D.; Doerr, J.A.; Hamilton, P.B. and Burmeister, H.R. (1975b): Egg production, shell thickness and other physiological parameters of laying hens affected by T-2 toxin. Appl. Microbial., 29: 641-645.

- Young, D.S. (2001): Effects of disease on clinical lab. Tests, $4^{\text {th }}$ Ed. AACC. 